MZ-TH/98-56

hep-ph/9901287

\title{
HERA Physics Beyond the Standard Model
}

\author{
H Spiesberger \\ Institut für Physik, Johannes-Gutenberg-Universität, D-55099 Mainz, Germany
}

\begin{abstract}
The prospects of physics beyond the standard model in deep inelastic scattering are reviewed, emphasizing some scenarios which attained attention after the observation of an excess of events with large momentum transfer at HERA用.
\end{abstract}

\section{Introduction}

After 6 years of running and major improvements of the electron proton collider machine, the experiments at HERA start to open a new focus of physics analyses looking at processes with cross sections of the order of $1 \mathrm{pb}$ and below. This is the typical value for cross sections at large values of Bjorken $x$ and momentum transfer $Q^{2}$, or more generally of processes with large transverse momenta. At the upper limit of the available centerof-mass energy, the cross sections for deep inelastic scattering are equally determined by both electromagnetic and purely weak interactions. Moreover, measurements are possible of rare standard model processes like the production of an additional gauge boson, or of radiative processes in neutral and charged current scattering. Other examples are the production of lepton pairs or multi-jet systems with large invariant masses. These low cross section processes provide a wealth of possibilities to look for deviations from the standard model predictions and constitute important backgrounds for searches for physics beyond the standard model [1, 2].

New physics may be found in a search for processes which are forbidden in the standard model or have tiny cross sections much below the level of $1 \mathrm{pb}$; some models of physics beyond the standard model predict such "gold-plated", backgroundfree signatures. However, more common is the situation that the cross section for conventional standard model processes are only slightly modified. The first task when searching for new physics is therefore to obtain a precise and detailed knowledge of standard model predictions. For deep inelastic scattering this task is twofold: on the one hand one has to provide precise parametrizations of parton distribution functions evolved in $Q^{2}$ according to next-to-leading order of QCD. On the other hand, the cross sections for hard lepton-quark and lepton-gluon subprocesses have to be known also at least to next-to-leading order. The theoretical tools needed to solve the first part of the task are well-established and the precision of parton distribution functions depends

† Talk presented at the 3rd UK Phenomenology Workshop on HERA Physics, September 1998, Durham 
mainly on the quality of experimental data [3]. On the other hand, NLO calculations for hard subprocesses, while continuously improving, have not yet reached a completely satisfactory status [4].

NLO calculations for inclusive scattering, i.e. $O\left(\alpha_{s}\right)$ corrections to the structure functions $\left(F_{L}, \Delta F_{3}\right.$ and, in the $\overline{\mathrm{MS}}$ scheme, $\left.\Delta F_{2}\right)$ are known since long [5]. Considerable progress has been made with respect to jet production in DIS and photoproduction [6], but $Z$ and $W$ exchange, relevant at large $Q^{2}$, is not taken into account in the available NLO Monte Carlo programs. Only recently, next-to-leading order corrections to the $W$ production $e p \rightarrow W+X$, including resolved contributions to photoproduction, have been reported [7]. Calculations for NLO corrections to the production of isolated photons are available for deep inelastic scattering [8] and for photoproduction [9] (see also [10]), but the transition region of small and large $Q^{2}$ has not yet been investigated. For other cases, like for example lepton pair production, NLO, sometimes even LO, calculations are still missing.

The demands for the precision of standard model predictions varies depending on

the size of the cross section. For inclusive measurements in deep inelastic scattering one requires rather precise measurements when searching for new physics. Specific final states, in particular when they contain one or two particles with high transverse energy, can be left at a precision of $O(10 \%)$. For "generic searches", where deviations from standard model predictions are looked for without referring to any specific expectations [4], a classification of "interesting" final states containing a high- $p_{T}$ charged lepton, large missing transverse momentum, or a high- $p_{T}$ jet, or any combination of them, would be helpful for a systematic investigation of experimental uncertainties.

The motivation to search for new physics at HERA has received a strong impetus by the observation of enhancements of cross sections at several places. The excess of events at large $x$ and large $Q^{2}$ in neutral and charged current scattering [11] has been discussed at lenght in the literature, see [12, 13] and references therein. Notably the occurence of events with an isolated muon and large missing transverse momentum at H1 [14] which are seemingly not a sign of $W$ production presents a challenge for the understanding of the experiments. Also at other experiments data suggest themselves as an indication for the appearance of new physics. I would like to mention only the high- $E_{T}$ dijets at Tevatron [15] and the cross section for $e^{+} e^{-} \rightarrow W^{+} W^{-}$at LEP2 which is too low at the highest $\sqrt{s}$ [16]. However, in the latter case, the experiments find a suppression, not an enhancement, and it seems more difficult to find a scenario which predicts the necessary amount of interference with the standard model. It is therefore more wide-spread to believe that this is a statistical fluctuation, but the same can be the origin of all the other observations as well. Probably the most strong hint for the presence of new physics is the experimental evidence for neutrino oscillations [17].

In the following, I selected some of the alternatives to standard model physics which, if realized in nature, have a good chance to be discovered at HERA. If not, HERA is expected to significantly contribute to setting limits on their respective model parameters. Other topics of interest are discussed in [1], 2, 18]. 


\section{The main alternatives}

Despite of the great success of the standard model, various conceptual problems provide a strong motivation to look for extensions and alternatives. Two main classes of frameworks can be identified among the many new physics scenarios discussed in the literature:

- Parametrizations of more general interaction terms in the Lagrangian like contact interactions or anomalous couplings of gauge bosons are helpful in order to quantify the agreement of standard model predictions with experimental results. In the event that deviations are observed, they provide a framework that allows to relate different experiments and cross-check possible theoretical interpretations. Being insufficient by themselves, e.g. because they are not renormalizable, parametrizations are expected to show the directions to the correct underlying theory if deviations are observed.

- Models, sometimes even complete theories, provide specific frameworks that allow a consistent derivation of cross sections for conventional and new processes. Examples are the two-Higgs-doublet extension of the standard model, grand unified theories and, most importantly supersymmetry with or without $\not R_{p}$-violation.

The following three examples attained most interest when the excess of large- $Q^{2}$ events at HERA was made public [12, 13. I will try to point out some of the open questions worth to be studied in future theoretical research.

\subsection{Contact interactions}

The contact interaction (CI) scenario relevant for HERA physics assumes that 4-fermion processes are modified by additional terms in the interaction Lagrangian of the form

$$
\mathcal{L}_{\mathrm{CI}}=\sum_{\substack{i, k=L, R \\ q=u, d, \cdots}} \eta_{i k}^{q} \frac{4 \pi}{\left(\Lambda_{i k}^{q}\right)^{2}}\left(\bar{e}_{i} \gamma^{\mu} e_{i}\right)\left(\bar{q}_{k} \gamma_{\mu} q_{k}\right) .
$$

Similar terms with 4-quark interactions would be relevant for new physics searches at the Tevatron and 4-lepton terms would affect purely leptonic interactions. In equation (回), as usual, only products of vector or axial-vector currents are taken into account since limits on scalar or tensor interactions are very stringent. Such terms are motivated in many extensions of the standard model as effective interactions after having integrated out new physics degrees of freedom like heavy gauge bosons, leptoquarks and others,

with masses beyond the production threshold. The normalization with the factor $4 \pi$ is reminiscent of models which predict CI terms emerging from strong interactions at a large mass scale $\Lambda$. Beyond their meaning as new physics effects, limits on the mass scale of contact interactions serve as an important means to quantify the agreement of experimental data with standard model predictions. 


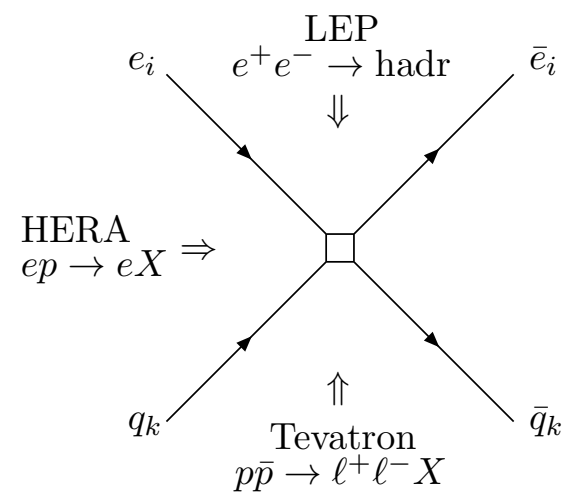

Figure 1. Schematic view of a contact interaction term.

Equation 1 predicts modifications of cross sections for 4-fermion processes in all channels as visualized in Fig. 1. Both enhancement or suppression are expected at the largest possible energies if the CI mass scale is large, depending on the helicity structure of the contact term and its sign $\eta_{i k}^{q}$. Due to the extremely high experimental precision, also atomic parity violation experiments are sensitive to parity-odd combinations of helicities. The important advantage of the contact term approach is that it provides a framework which can be applied to all presently running high-energy experiments. The contact term approach relates predictions for DIS at HERA with hadron production in electron positron annihilation and Drell-Yan production at the Tevatron.

\begin{tabular}{llllllll}
\hline & \multicolumn{3}{c}{ Drell-Yan } & \multicolumn{2}{c}{$e p \rightarrow e X$} & \multicolumn{2}{c}{$e^{+} e^{-} \rightarrow$ hadrons } \\
\cline { 2 - 8 } & $\mathrm{CDF}(e e, \mu \mu)$ & $\mathrm{D} 0(e e)$ & H1 & ZEUS & Aleph & L3 & Opal \\
\hline$V V+$ & 3.5 & 4.7 & 4.5 & 4.9 & 4.0 & 3.9 & 4.1 \\
$V V-$ & 5.2 & 5.8 & 2.5 & 4.6 & 5.2 & 5.0 & 5.7 \\
$A A+$ & 3.8 & 4.6 & 2.0 & 2.0 & 5.6 & 5.6 & 6.3 \\
$A A-$ & 4.8 & 5.3 & 3.8 & 4.0 & 3.7 & 3.5 & 3.8 \\
\hline
\end{tabular}

Table 1. Typical limits (in GeV) for the mass scale of CI terms from Tevatron, HERA and LEP for pure vector-vector and axial-vector-axial-vector type with positive or negative sign relative to the standard model interactions [19].

Table 1 gives a selection of recent limits on CI mass scales as reported at the 1998 summer conferences (limits for other combinations of helicities are available as well [19]). The numbers in this table show that all present high-energy experiments have achieved limits in a very similar mass range despite of their different center-ofmass energies. Consequently, with a signal at HERA one should expect visible effects at LEP2 and at the Tevatron. In the case of the observation of deviations from the standard model predictions, the combination of results obtained in different experiments and from measurements with polarized beams [20] will be helpful to identify the helicity structure of contact interaction terms. 


\subsection{Leptoquarks}

Leptoquarks appear in extensions of the standard model involving unification, technicolor, compositeness, or $R$-parity violating supersymmetry. In addition to their couplings to the standard model gauge bosons, leptoquarks have Yukawa-type couplings to lepton-quark pairs which allow their resonant production in $e p$ scattering. Their phenomenology in view of the observed excess of large- $x$, large- $Q^{2}$ events at HERA has been discussed extensively in the literature (《13 and references therein). The generally adopted framework described in Ref. [21] is based on the assumption that the Yukawa interactions of leptoquarks should have the following properties:

- renormalizability

- $S U(3) \times S U(2) \times U(1)$ symmetry

- conservation of baryon and lepton number

- chirality of the couplings

- couplings exist only to one fermion generation

- no other interactions and/or particles exist

Dropping one of the first two of these assumptions would lead to severe theoretical problems; the other properties are dictated by phenomenology. One would certainly not like to give up assumption 3 since this avoids rapid proton decay. The chirality of couplings is necessary in order to escape the very strong bounds from leptonic pion decays and assumption 5 is a consequence of limits on FCNC processes. The last assumption is made for simplicity only; it seems to be rather unlikely than realistic.

These assumptions lead to a rather restricted set of allowed states and their branching fractions $\beta$ to a charged lepton final state can only be $1,0.5$, or 0 . Those states which are interesting for HERA phenomenology have $\beta=1$ and are excluded by Tevatron bounds which require masses above $242 \mathrm{GeV}$ [22].

The leptoquark scenario might remain interesting if it is possible to generalize the approach by relieving one or more of the above assumptions [23], notably the last one of the list. The Tevatron mass bounds are avoided if it was possible to adjust the branching ratios in the range $0.3 \lesssim \beta \lesssim 0.7$ [12, 23]. In Ref. [24] a scenario was proposed where two leptoquark states show mixing induced by coupling them to the standard model Higgs boson. Alternatively, interactions to new heavy fields might exist that, after integrating them out, could lead to leptoquark Yukawa couplings as an effective interaction [25], bypassing this way renormalizability as a condition since this is assumed to be restored at higher energies. In the more systematic study of Ref. [23], LQ couplings arise from mixing of standard model fermions with new heavy fermions with vector-like couplings and taking into account a coupling to the standard model Higgs. Up to now, no attempt was made to study in a systematic way the possibility of relieving the assumption that no intergenerational couplings should exist (see however Ref. [25, 26]). The most interesting extension of the generic leptoquark scenario is, however, $R_{p}$-violating supersymmetry which is discussed in the next subsection. 


\section{3. $R_{p}$-violating supersymmetry}

The Lagrangian of a supersymmetric version of the standard model may contain a superpotential of the form

$$
\begin{aligned}
& W_{R_{p}}=\quad \lambda_{i j k} L_{i} L_{j} E_{k}^{c} \quad \text { IL } \\
& +\lambda_{i j k}^{\prime} L_{i} Q_{j} D_{k}^{c} \quad \Psi \quad \text { (includesLQ }- \text { like couplings) } \\
& +\lambda_{i j k}^{\prime \prime} U_{i}^{c} D_{j}^{c} D_{k}^{c} \quad \not B
\end{aligned}
$$

which violates lepton or baryon number conservation as indicated. Imposing symmetry under $R$-parity (defined as $R_{p}=(-1)^{3 B+L+2 S}$ ) forbids the presence of $W_{R_{p}}$. The resulting phenomenology has been searched for at all present high energy experiments and HERA may set interesting limits which are complementary to those obtained at Tevatron [1]. Future experiments at the LHC will extend the search limits for $R_{p^{-}}$ conserving supersymmetry considerably.

The present limits on the proton life-time do not forbid interactions of the form $L_{i} Q_{j} D_{k}^{c}$ proportional to $\lambda_{i j k}^{\prime}$ provided the $\lambda_{i j k}^{\prime \prime}$ are chosen to be zero at the same time. This makes squarks appear as leptoquarks which can be produced on resonance in lepton-quark scattering. In contrast to the generic leptoquark scenarios described above, $R_{p}$-conserving decays of squarks lead to a large number of interesting and distinct signatures (see Ref. [27] and references therein). Characteristically one expects multilepton and multi-jet final states. The branching ratios can be adjusted so as to avoid the strict mass limits from Tevatron.

Most of the analyses done so far assume that only one of the couplings $\lambda_{i j k}^{\prime}$ is non-zero and only one squark state is in reach. A more general scenario with two light squark states has been considered in Ref. [28] where it was shown that $\tilde{t}_{L}-\tilde{t}_{R}$ mixing would lead to a broader $x$ distribution than expected for single-resonance production. The possibility of having more than one $\lambda_{i j k}^{\prime} \neq 0$ was noticed in Ref. [29] and deserves more theoretical study.

$R_{p}$-violating supersymmetry has also played a role in the search for explanations of the observation of a large number of events with an isolated $\mu$ and missing transverse momentum [14]. Events of this kind can originate from $W$ production followed by the decay $W \rightarrow \mu \nu_{\mu}$; their observed number is, however, larger than expected and their kinematical properties are atypical for $W$ production. An explanation in terms of anomalous $W W \gamma$ couplings additionally has to face limits from Tevatron and LEP2 and leaves the question open why a similar excess of events is not seen in $e+p_{T}$ events.

The observation of $\mu+\not p_{T}$ events could find an explanation in $R_{p}$-violating scenarios if it is assumed that a stop is produced on-resonance at HERA. Figures 2 and 3 show examples for some of the possibilities. The process $e d \rightarrow \tilde{t} \rightarrow \mu d^{k}$ (Fig. 国a) which predicts $\mu$ but no large $p_{T}$ in the final state requires two different non-zero $\lambda^{\prime}$ couplings. The relevant product $\lambda_{1 j 1}^{\prime} \lambda_{2 j k}^{\prime}$ would induce flavor changing neutral currents and is therefore limited to unreasonably small values for $1^{\text {st }}$ and $2^{\text {nd }}$ generation quarks in the final state [30]. The scenario shown in Fig. 2b [31] requires a relatively light $b$

squark, $m_{\tilde{b}} \lesssim 120 \mathrm{GeV}$, and some fine-tuning in order to avoid too large effects on $\Delta \rho$ in 
electroweak precision measurements. It could be identified by the simultaneous presence of multi-jet final states with $p_{T}$ from hadronic decays of the $W$. Also the cascade decay shown in Fig. 3a 32 involving $R_{p}$-violation only for the production of the $\tilde{t}$ resonance, not for its decay, seems difficult to be achievable since it requires both a light chargino and a long-lived neutralino. This, as well as the even more speculative process shown in Fig. 3b [33] which requires $R_{p}$-violation in the $L_{i} L_{j} E_{k}^{c}$ sector $\left(\lambda_{i j k} \neq 0\right)$ as well, can be checked from the event kinematics: assuming a value for the mass of the decaying $\tilde{t}$, the recoil mass distribution must cluster at a fixed value, the chargino mass. A more detailed discussion of the $\mu+p_{T}$ events and their possible theoretical origin can be found in 34 .

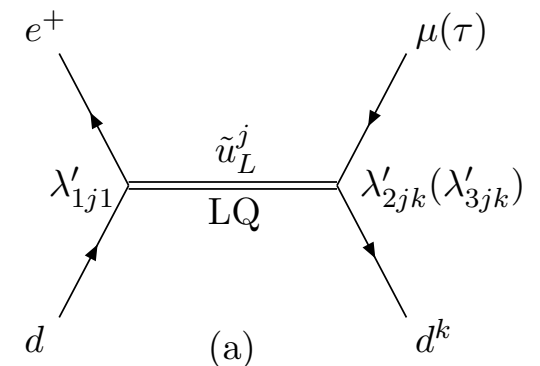

(a)

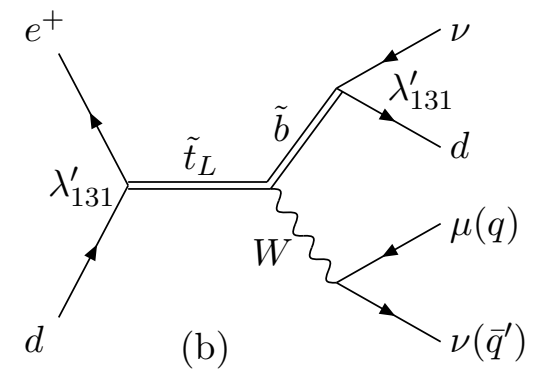

(b)

Figure 2. Possible decays of squarks produced in $e^{+} d$ scattering with $R_{p^{-}}$violating couplings leading to isolated $\mu+$ jet final states: (a) $\tilde{u}_{L}^{j} \rightarrow \mu d_{k}$ through $\lambda_{2 j k}^{\prime} \neq 0$; (b) $\tilde{t} \rightarrow \tilde{b} W$ followed by $\tilde{b} \rightarrow \nu d$ via $\lambda_{131}^{\prime} \neq 0$ and $W \rightarrow \mu^{+} \nu_{\mu}$ or $W \rightarrow 2$ jets [31].

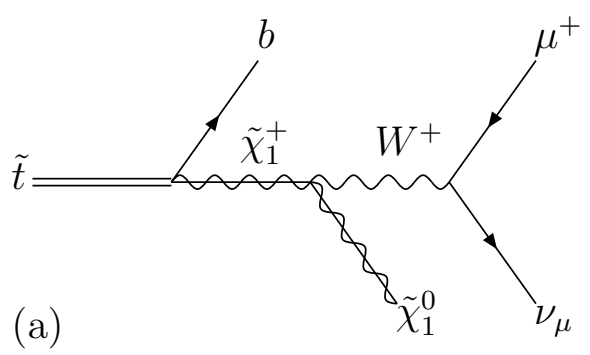

Figure 3. Possible decay chains of the stop leading to isolated muon + jet + missing $p_{T}: \tilde{t} \rightarrow b \tilde{\chi}_{1}^{+}$followed by (a) $\tilde{\chi}_{1}^{+} \rightarrow \tilde{\chi}_{1}^{0} \mu^{+} \nu_{\mu}$ [32]; (b) $\tilde{\chi}_{1}^{+} \rightarrow \nu_{\ell} \mu^{+} \nu$ [33].

\section{Concluding remarks}

The search for new physics effects relies in many cases on trustworthy predictions from the standard model, in particular when generic searches look for "interesting" final states without having at hand a specific model that tells the experimenter what and where to look for precisely. New physics will always, if at all, show up at the frontier of the experiments, i.e. at the largest energies or transverse momenta where cross sections are smallest and experimental problems most severe. It is therefore a mandatory though nontrivial task to combine the information from as many as possible different experiments. In order to enhance the statistical significance and reduce the probability that experimental deficiencies lead to wrong interpretations, also experiments which did 
not obtain the most stringent limits are important. The experiments at HERA are therefore guaranteed to contribute to the search for new physics.

\section{References}

[1] Future Physics at HERA, Proceedings of the Workshop 1995/96 Eds Ingelman G, DeRoeck A and Klanner R (DESY Hamburg) Vol 1 p 237

[2] Waters D S 1998 and other contributions to These proceedings

[3] Stirling W J 1998 These proceedings

[4] Krasny M W and Spiesberger H 1998 These proceedings

[5] Altarelli G, Ellis R K and Martinelli G 1978 Nucl. Phys. B143 521; Körner J, Mirkes E and Schuler G 1989 Int. J. Mod. Phys. A4 1781

[6] Graudenz D 1998 These proceedings; Hadig T 1998 These proceedings; Butterworth J, Maxfield S, Pötter B and Sinclair L 1998 These proceedings; Pötter B and Seymour M 1998 These proceedings

[7] Nason P, Rückl R and Spira M 1998 in preparation

[8] Michelsen D, Kramer G and Spiesberger H, 1998 Eur. Phys. J. C5 293

[9] Aurenche P et al. 1984 Z. Phys. C24 309, 1989 Phys. Rev. D39 3275, 1993 Z. Phys. C56 589 ; Gordon L E 1998 Phys. Rev. D57 235, Gordon L E, Vogelsang W 1995 Phys. Rev. D52 58, Gordon L E, Storrow J K 1994 Z. Phys. C63 581

[10] Bussey P and Fontannaz M 1998 These proceedings

[11] H1 Collaboration Adloff C et al 1997 Z. Phys. C74 191; ZEUS Collaboration Breitweg J et al 1997 Z. Phys. C74 207

[12] Altarelli G 1998 Proc. Conference on Supersymmetries in Physics (SUSY 97) Nucl. Phys. B Proc. Suppl. 623

[13] Rückl R and Spiesberger H 1997 Proc. Workshop on Physics Beyond the Standard Model: Beyond the desert Tegernsee Germany p 304 (hep-ph/9711359) ; Ringberg Workshop on New Trends in HERA Physics, Ringberg Castle Tegernsee 1997 p 113 (hep-ph/971032才)

[14] H1 Collaboration Adloff C et al 1998 Eur. Phys. J. C5 575

[15] CDF collaboration Abe F et al 1996 Phys. Rev. Lett. 77 438; Phys. Rev. Lett. 775336

[16] Karlen D 1998 Proc. ICHEP98 Vancouver 1998

[17] Takita M 1998 Proc. ICHEP98 Vancouver 1998

[18] Schrempp F 1998 These proceedings

[19] Treille D 1998 Proc. ICHEP98 Vancouver 1998

[20] Kalinowski J, Spiesberger H and Virey J M 1998 hep-ph/9812517 and These proceedings

[21] Buchmüller W, Rückl R and Wyler D 1987 Phys. Lett. B191 442

[22] Leptoquark Limit Combination Working Group (for the CDF and D0 Collaborations) 1998 hep$e x / 9810015$

[23] Hewett J L and Rizzo T G 1998 Phys. Rev. D58 55005

[24] Babu K S, Kolda C and March-Russell J 1997 Phys. Lett. B408 261

[25] Altarelli G, Giudice G F and Mangano M L 1997 hep-ph/9705287

[26] Kunszt Z and Stirling W J 1997 Z. Phys. C 75453

[27] Perez E, Sirois Y and Dreiner H 1996 Future Physics at HERA, Proceedings of the Workshop 1995/96 DESY Hamburg hep-ph/9703444 Dreiner H 1997 hep-ph/9707435

[28] Kon T, Kobayashi T 1997 Phys. Lett. B409 265

[29] Belyaev A S and Gladyshev A V $1998 h e p-p h / 980754$ \}

[30] Davidson S, Bailey D and Campbell B 1994 Z. Phys. C61 613

[31] Kon T, Matsushita T and Kobayashi T 1997 Mod. Phys. Lett. A12 3143

[32] Kon T, Kobayashi T and Kitamura S 1996 Phys. Lett. B376 227

[33] Kalinowski J, Rückl R, Spiesberger H and Zerwas P 1997 DESY internal note unpublished

[34] Diaconu C, Kalinowski J, Matsushita T, Spiesberger H and Waters D S 1998 These proceedings 